\title{
AUTOMATISIERTE FAHRZEUGE IM STRAßENVERKEHR
}

\author{
Georg Borges ${ }^{1}$
}

\begin{abstract}
Hochautomatisierte Fahrzeuge sind ein wichtiges und interessantes Beispiel für die Herausforderungen, die sich durch mit künstlicher Intelligenz ausgestattete Systeme für das Recht ergeben: Bei Kraftfahrzeugen handelt es sich um grenzüberschreitend eingesetzte, international vertriebene Produkte, die aufgrund des erheblichen Gefahrenpotentials einer intensiven rechtlichen Regelung unterliegen. Der Beitrag erörtert anhand der Anforderungen an die Zulassung hochautomatisierter Fahrzeuge zum Straßenvekehr die besonderen Herausforderungen, die sich durch künstliche Intelligenz für die Regulierung von Technologie ergeben.
\end{abstract}

\section{Inhaltsverzeichnis}

A. Einführung................................................................................................................ 322

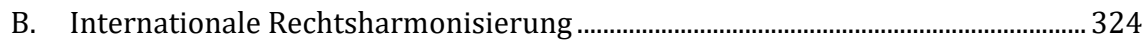

C. Dynamisierung des Rechts durch Verweis auf technische Standards ..................326

I. Betriebserlaubnis und technische Standards.....................................................326

1. Konformität mit den technischen Bedingungen des Anhangs 5 ............ 327

2. Vereinbarkeit mit technischen Normen auf Grundlage internationaler Übereinkommen ................................................................ 327

II. Verweis auf technische Standards in der Typgenehmigung........................... 329

III. Koordinierung des WÜ und des europäischen Rechts mit den UN/ ECE-Regelungen. 331

IV. Zwischenergebnis 332

1 Der Autor ist Universitätsprofessor für Bürgerliches Recht, Rechtsinformatik, deutsches und internationales Wirtschaftsrecht sowie Rechtstheorie und geschäftsführender Direktor des Instituts für Rechtsinformatik an der Universität des Saarlandes. 
D. Strukturelle Herausforderungen für das Recht durch Künstliche Intelligenz.......333

I. Autonomes Verhalten von Fahrzeugen als Herausforderung..........................333

II. Das traditionelle Erfordernis eines menschlichen Fahrzeugführers.............333

III. Künstliche Intelligenz als Herausforderung für das System der UN/ ECE-Regelungen

IV. Herstellererklärung vs. Typgenehmigung?

V. Notwendigkeit der Fortentwicklung des Rechtsrahmens für automatisierte Fahrzeuge

E. Fazit

\section{A. EINFÜHRUNG}

Künstliche Intelligenz und sog. autonome Systeme, die aufgrund der Ausstattung mit künstlicher Intelligenz eigenständig Entscheidungen treffen und ausführen können, stellen das Recht vor gewaltige Herausforderungen, die in jüngster Zeit Gegenstand einer ungeheuer dynamischen Diskussion in zahlreichen Rechtsgebieten sind. Ein Schwerpunkt dieser Diskussion liegt, angesichts der praktischen Bedeutung und der Anschaulichkeit der Fragen zweifellos zu Recht, auf dem automatisierten Fahren. ${ }^{2}$

Eine zentrale Frage betrifft die rechtliche Regelung der Nutzung hoch- und vollautomatisierter Fahrzeuge. Die Voraussetzungen für die Teilnahme am öffentlichen Straßenverkehr, insbesondere mit Kraftfahrzeugen sind rechtlich stark reguliert. Dabei wird, nach einem jedenfalls in den meisten Rechtsordnungen weltweit verwendeten Regelungskonzept, der Betrieb eines Kraftfahrzeugs, im öffentlichen Straßenverkehr von einer Zulassung des jeweiligen Fahrzeugs zum Straßenverkehr abhängig gemacht. So bestimmt etwa das deutsche Recht in $\S 1$ Abs. 1 S. 1 des Straßenverkehrsgesetzes

2 Als Beispiel sei hier das soeben in zweiter Auflage erschienene Handbuch OPPERMAN/STENDERVoRWACHS (Hrsg.), Autonomes Fahren, 2020, genannt, das auf etwa 500 Seiten zahlreiche Rechtsgebiete adressiert. Großen Einfluss in der Praxis hatte wohl die Untersuchung von GASSer, T., Arzt, C., Ayoubi, M., BArtels, A., BÜrkle, L., Eier, J., Flemisch, F., HÄcker, D., Hesse, T., Huber, W., Lotz, C., Maurer, M., Ruth-Schumacher, S., SchWARZ, J., Vogt, W.: Rechtsfolgen zunehmender Fahrzeugautomatisierung - Gemeinsamer Schlussbericht der Projektgruppe, Berichte der Bundesanstalt für Straßenwesen, Bergisch-Gladbach (2012). 
(StVG), dass Kraftfahrzeuge, die auf öffentlichen Straßen in Betrieb gesetzt werden sollen, von der zuständigen Behörde (Zulassungsbehörde) zum Verkehr zugelassen sein müssen.

Die Zulassung wird dabei an die Erfüllung bestimmter Anforderungen an das Fahrzeug geknüpft. Die wesentliche Voraussetzung der Zulassung ist das Vorliegen einer fahrzeugbezogenen Genehmigung (§ 1 Abs. 1 S. 2 StVG), der sog. Betriebserlaubnis. ${ }^{3}$ Häufig erfordert die Zulassung darüber hinaus, etwa im deutschen Recht, die Erfüllung weiterer Anforderungen, etwa das Bestehen einer Haftpflichtversicherung für das Fahrzeug. ${ }^{4}$ Daneben - und weitgehend unabhängig davon ${ }^{5}$ - gelten Anforderungen an das Verhalten im Straßenverkehr, etwa die Beachtung von Verkehrsregeln.

Bei Kraftfahrzeugen stellen sich seit jeher besondere Anforderungen an die rechtliche Regelung, die zum einen den grenzüberschreitenden Einsatz von Fahrzeugen und das Bedürfnis nach internationalem Vertrieb der Fahrzeuge berücksichtigen muss und zum anderen der dynamischen technischen Entwicklung Rechnung zu tragen hat.

Bei Fahrzeugen mit automatisierten Fahrfunktionen, insbesondere bei hoch- und vollautomatisierten Fahrzeugen, stellen sich in beiden Bereichen erhebliche Herausforderungen. Fahrzeuge werden in Bezug auf den Automatisierungsgrad heute meist anhand des SAE Standard J3016_201806 6 eingestuft, der sechs Automatisierungsstufen (sog. Level) von Stufe o (keine Fahrautomatisierung) bis zu Stufe 5 (vollständige Fahrautomatisierung) unterscheidet. Da die Besonderheiten der verschiedenen Automatisierungsstufen nicht im Fokus dieser Untersuchung stehen, wird nachfolgend zur Vereinfachung von hochautomatisierten Fahrzeugen (Stufe 4) gesprochen, die technisch

$3 \S 1$ Abs. 1 S. 2 StVG lautet: «Die Zulassung erfolgt auf Antrag des Verfügungsberechtigten des Fahrzeugs bei Vorliegen einer Betriebserlaubnis, Einzelgenehmigung oder EG-Typgenehmigung durch Zuteilung eines amtlichen Kennzeichens.»

4 § 1 Abs. 1 S. 2 StVG i.V.m. § 3 Abs. 1 S. 2 der Fahrzeugzulassungsverordnung (FZV).

5 Zur notwendigen Verknüpfung von Zulassung und Verkehrsregeln beim hochautomatisierten Fahren siehe unten D.III.

6 SAE International, Taxonomy and Definitions for Terms Related to Driving Automation Systems for On-Road Motor Vehicles, Standard J3016, 2018. 
zum vollständig selbstständigen Fahren in der Lage sind und, anders als bei Fahrzeugen der Stufe 3, nicht auf einen Fahrzeugbenutzer angewiesen sind, der jederzeit zur Übernahme der Fahrzeugsteuerung bereit ist. Die hier erörterten Rechtsfragen stellen sich freilich bei vollautomatisierten Fahrzeugen der Stufe 5 in gleicher Weise, ähnlich auch bei Fahrzeugen niedrigerer Automatisierungsstufen.

Der Rechtsrahmen der Teilnahme am Straßenverkehr hat auf diese Herausforderungen spezifische Antworten gefunden: Der Dynamik der technischen Entwicklung begegnet das Recht durch Verweis auf technische Standards (dazu unten C.II.). Der Bedarf nach internationaler Harmonisierung des Rechts wird durch ein Konzept der Vereinheitlichung und der Anerkennung von Anforderungen im Bereich der Zulassung erfüllt, das vor allem in jüngster Zeit durch eine inhaltliche Harmonisierung der technischen Standards realisiert wird.

Diese Instrumente sind bei hochautomatisierten Fahrzeugen von großer Bedeutung. Darüber hinaus zeigt sich nicht zuletzt am Beispiel des hochautomatisierten Fahrens, dass der Betrieb von Systemen, die mit Hilfe künstlicher Intelligenz eigenständig operieren, eine Neukonzeption des Rechtsrahmens erfordert, die über die bekannten Instrumente hinausgeht.

Nachfolgend werden zunächst die Instrumente der Internationalisierung und Dynamisierung des Rechtsrahmens am Beispiel der Zulassung von Fahrzeugen zum Straßenverkehr erörtert und sodann einige der Herausforderungen für den Rechtsrahmen der Zulassung aufgezeigt.

\section{B. INTERNATIONALE RECHTSHARMONISIERUNG}

Die internationale Harmonisierung der rechtlichen Anforderungen an den Betrieb von Fahrzeugen erfolgt vor allem durch das Völkerrecht. Das zentrale völkerrechtliche Instrument ist das Wiener Übereinkommen über den Straßenverkehr von 1968 (WÜ)7, das 1977 in Kraft getreten ist. Derzeit sind 8o Nationen Vertragsstaaten des

7 Übereinkommen vom 8. November 1968 über den Straßenverkehr Es handelt sich um zwei verschiedene Übereinkommen mit einer unterschiedlichen Anzahl an Mitgliedstaaten. Vorliegend kann nur das Übereinkommen über den Straßenverkehr gemeint sein. 
Übereinkommens, darunter auch Deutschland, Österreich und zahlreiche weitere europäische Staaten, nicht aber etwa die USA und China. ${ }^{8}$

Das WÜ wurde zuletzt 2016 geändert und gilt heute in der revidierten Fassung vom 23. März 2016. ${ }^{9}$ Diese Änderung geht auf einen Antrag von Belgien, Deutschland, Frankreich, Italien und Österreich zurück, der im März 2014 von der Working Party on Road Traffic Safety (WP.1) der UN/ECE angenommen wurde. ${ }^{10}$

Das Übereinkommen adressiert wesentliche Regelungen für die Teilnahme am Straßenverkehr mit Kraftfahrzeugen. Von zentraler Bedeutung sind insbesondere das Kapitel 2, das wesentliche Grundsätze zum Verhalten im Straßenverkehr regelt, das Kapitel 3 zur Zulassung von Kraftfahrzeugen zum Straßenverkehr und das Kapitel 4 zum Führer von Kraftfahrzeugen, das vor allem das Erfordernis eines Führerscheins enthält.

Das WÜ verpflichtet die Vertragsstaaten insbesondere dazu, die innerstaatlichen Verkehrsregeln in Übereinstimmung mit Kapitel 2 zu bringen (Art. 3 Abs. 1 WÜ), die Regeln für die technischen Anforderungen an Kraftfahrzeuge in Übereinstimmung mit dem Anhang 5 des WÜ zu gestalten (Art. 3 Abs. 2 WÜ) und durch andere Vertragsstaaten zum Verkehr zugelassene Kraftfahrzeuge zur Teilnahme am Straßenverkehr zuzulassen, soweit diese die Anforderungen des WÜ erfüllen (Art. 3 Abs. 3 WÜ).

8 Eine Liste der Vertragsstaaten ist abrufbar unter https://treaties.un.org/Pages/ViewDetailsIII.aspx?src=IND\&mtdsg_no=XI-B-19\&chapter=11\&Temp=mtdsg3\&clang=_en (abgerufen am 13.01.2020).

9 Vgl. die Bekanntmachung der Annahme des Änderungsvorschlages, abrufbar unter https://treaties.un.org/doc/Publication/CN/2015/CN.529.2015.Reissued.06102015-Eng.pdf; der Volltext der Änderungen ist abrufbar unter http://www.unece.org/fileadmin/DAM/ trans/doc/2014/wp1/ECE-TRANS-WP1-145e.pdf(abgerufen am 13.01.2020).

10 Economic Commission for Europe/Working Party on Road Traffic Safety, Report of the sixtyeighth session of the Working Party on Road Traffic Safety (Geneva, 24-26 March 2014), ECE/TRANS/WP.1/145, sub. V. A., S. 5 (abrufbar unter: https://www.unece.org/filead$\mathrm{min} / \mathrm{DAM} /$ trans/doc/2014/wp1/ECE-TRANS-WP1-145e.pdf)(abgerufen am 13.01.2020). 


\section{DYNAMISIERUNG DES RECHTS DURCH VERWEIS AUF TECHNISCHE STANDARDS}

Eine traditionelle Herausforderung der Regulierung i.S. der Gestaltung des rechtlichen Rahmens von Technologie betrifft das Erfordernis, bei der Formulierung rechtlicher Anforderungen mit der mitunter rasanten technischen Entwicklung Schritt zu halten.

Diese Herausforderung wird in Bezug auf die Teilnahme von Fahrzeugen am Straßenverkehr durch den Verweis auf technische Normen gelöst. Dies gilt traditionell für die Zulassung von Fahrzeugen zum Straßenverkehr, in jüngster Zeit aber auch für Anforderungen an das Verhalten im Straßenverkehr.

\section{Betriebserlaubnis und technische Standards}

Die Zulassung eines Fahrzeuges zum Straßenverkehr ist, wie dargestellt, die zentrale Voraussetzung dafür, dass ein Kraftfahrzeug im Straßenverkehr in Betrieb gesetzt werden darf.

Das Wiener Übereinkommen regelt das eigentliche Zulassungsverfahren nicht, normiert aber in Kapitel III allgemeine Bedingungen für die Zulassung von Kraftfahrzeugen. wesentlichen materiellen Anforderungen des WÜ an Fahrzeuge sind nach dessen Art. 39 Abs. 1 die Erfüllung bestimmter technischer Anforderungen sowie die Betriebssicherheit.11

In Bezug auf die Sicherstellung der Betriebssicherheit hat sich das System der Betriebserlaubnis als separates Verfahren zur Feststellung der Betriebssicherheit eines Fahrzeugs bzw. eines Fahrzeugtyps etabliert (dazu sogleich II.). Hinsichtlich der technischen Anforderungen enthält das WÜ, vor allem in Bezug auf hochautomatisierte Fahrzeuge, zwei unterschiedliche Regelungskonzepte.

11 Art. 39 Abs. 1 WÜ lautet: «Jedes Kraftfahrzeug, jeder Anhänger und alle miteinander verbundenen Fahrzeuge im internationalen Verkehr müssen dem Anhang 5 entsprechen. Sie müssen ferner betriebssicher sein. Sind diese Fahrzeuge mit Systemen, Teilen oder Ausrüstungsgegenständen ausgerüstet, die den technischen Vorschriften bezüglich Bauweise, Montage und Benutzung nach Maßgabe der in Absatz $5^{\text {bis }}$ von Artikel 8 dieses Übereinkommens erwähnten internationalen Rechtsvorschriften entsprechen, so gelten sie mit dem Anhang 5 als konform.» 


\section{Konformität mit den technischen Bedingungen des Anhangs 5}

Nach dem traditionellen Regelungskonzept des WÜ ist die Beachtung der technischen Bedingungen des Anhangs 5 von zentraler Bedeutung. So müssen die Vertragsstaaten, wie dargestellt, nach Art. 3 Abs. 2 WÜ sicherstellen, dass die Regeln des innerstaatlichen Rechts für Kraftfahrzeuge mit den obligatorischen technischen Bedingungen des Anhangs 5 übereinstimmen. Damit sind die Vertragsstaaten verpflichtet, die Anforderungen an die Zulassung von Kraftfahrzeugen zum Straßenverkehr an die Anforderungen des Anhangs 5 anzupassen. ${ }^{12}$ Weiterhin ist nach Art. 39 Abs. 1 S. 1 WÜ im internationalen Verkehr erforderlich, dass die Anforderungen des Anhangs 5 erfüllt sind. Die Konformität mit Anhang 5 ist damit die zentrale Voraussetzung sowohl für die Zulassung zum Straßenverkehr nach innerstaatlichem Recht der Vertragsstaaten als auch für die Zulassung zum internationalen Verkehr.

Anhang 5 des Übereinkommens enthält für zahlreiche Fahrzeugelemente bestimmte technisch-funktionale Vorgaben. ${ }^{13}$

\section{Vereinbarkeit mit technischen Normen auf Grundlage internationaler Übereinkommen}

Das WÜ enthält neben dem Verweis auf die Vorgaben des Anhangs 5 noch ein zweites System zur Erfüllung technischer Anforderungen, das auf die Normen internationaler Übereinkommen, insbesondere auf die sog. UN/ECE-Normen, verweist. Dieses System beruht auf Art. 8 Abs. $5^{\text {bis }}$ WÜ und Art. 39 Abs. 1 S. 3 WÜ, die mit Wirkung zum 23.3.2016 in das Übereinkommen eingefügt wurden. ${ }^{14}$ Nach Art. 39 Abs. 1 S. 3 WÜ gelten Fahrzeuge als mit Anhang 5 konform, soweit sie den technischen Vorschriften

12 V. Bodungen/Hoffmann, SVR 2016, 41, 43; LuTZ, in: HilgEndoRf/HötitZSCH/LuTZ (Hrsg.), Rechtliche Aspekte automatisierter Fahrzeuge - Beiträge zur 2. Würzburger Tagung zum Technikrecht im Oktober 2014, 2015, S. 33, 34.

13 BundeSAmt FÜR STRASSEN (ASTRA), Konzeptbericht «Führerlose Fahrzeuge - Rechtlicher Anpassungsbedarf» v. 22.4.2016, S. 15; s. ferner ARZT/RuTH-SCHUMACHER, NZV 2017, 57, 62; BEWERSDORF, NZV 2003, 266, 269 f.; v. BodUnGEN/HOFFMANN, SVR 2016, 41, 43.

14 Vgl. die Bekanntmachung der Annahme des Änderungsvorschlages, abrufbar unter https://treaties.un.org/doc/Publication/CN/2015/CN.529.2015.Reissued.06102015-Eng.pdf; der Volltext der Änderungen ist abrufbar unter http://www.unece.org/fileadmin/DAM/trans/ doc/2014/wp1/ECE-TRANS-WP1-145e.pdf (abgerufen am 13.01.2020). 
der in Art. 8 Abs. $5^{\text {bis } W U ̈ ~ e r w a ̈ h n t e n ~ « i n t e r n a t i o n a l e n ~ R e c h t s v o r s c h r i f t e n » ~ e n t s p r e-~}$ chen.

Bei den in Art. 8 Abs. $5^{\text {bis }}$ und Art. 39 Abs. 1 S. 3 WÜ genannten «internationalen Rechtsvorschriften» handelt es sich einerseits um die sog. UN/ECE-Normen sowie andererseits um die sog. «Globalen Technischen Vorschriften» der UN, 15 mithin die technischen Normungen auf Grundlage des sog. GTR-Abkommens. ${ }^{16}$

UN/ECE-Regelungen beruhen auf dem sog. «Fahrzeugteileübereinkommen» oder «UN/ECE-Übereinkommen», das 1958 in Genf beschlossen ${ }^{17}$ und seither zweimal geändert wurde. Es wird in der Revision 02 von 1995 auch oft als «Geändertes Übereinkommen» bezeichnet. Dem Übereinkommen gehören insg. 54 Parteien, überwiegend aus Europa, an. Japan ist Vertragsstaat, nicht aber etwa China sowie die USA. Die Europäische Union ist dem Übereinkommen 1997 beigetreten. ${ }^{18}$ Auf Grundlage des Übereinkommens, das auf die Schaffung einheitlicher technischer Vorschriften für Kraftfahrzeuge abzielt, ${ }^{19}$ wurden bereits fast 150 verschiedene Regelungen mit technischen

15 Siehe die erläuternde Fußnote zu Art. 8 Abs. $5^{\text {bis } W U ̈: ~}$

«Die Regelungen der Vereinten Nationen im Anhang des in Genf am 20. März 1958 beschlossenen «Übereinkommens über die Annahme einheitlicher technischer Vorschriften für Radfahrzeuge, Ausrüstungsgegenstände und Teile, die in Radfahrzeuge(n) eingebaut und/oder verwendet werden können, und die Bedingungen für die gegenseitige Anerkennung von Genehmigungen, die nach diesen Vorschriften erteilt wurden».

Die im Rahmen des in Genf am 25. Juni 1998 beschlossenen «Übereinkommens über die Festlegung globaler technischer Regelungen für Radfahrzeuge, Ausrüstungsgegenstände und Teile, die in Radfahrzeuge(n) eingebaut und/oder verwendet werden können» ausgearbeiteten globalen technischen Regelungen der Vereinten Nationen.»

16 Vereinbarung über die Festlegung globaler technischer Vorschriften für Radfahrzeuge, Ausrüstung und Teile, die an Radfahrzeugen angebracht und/oder verwendet werden können vom 25. Juni 1998, abrufbar unter: https://www.unece.org/fileadmin/DAM/trans/main/wp29/ wp29wgs/wp29gen/wp29glob/tran132.pdf.

Vgl. Fn. 15.

18 Beschluss 97/836/EG des Rates vom 27. November 1997 über den Beitritt der Europäischen Gemeinschaft zu dem Übereinkommen der Wirtschaftskommission für Europa der Vereinten Nationen über die Annahme einheitlicher technischer Vorschriften für Radfahrzeuge, Ausrüstungsgegenstände und Teile, die in Radfahrzeuge(n) eingebaut und/oder verwendet werden können, und die Bedingungen für die gegenseitige Anerkennung von Genehmigungen, die nach diesen Vorschriften erteilt wurden («Geändertes Übereinkommen von 1958», ABl. EG Nr. L 346 vom 17.12.1997, S. 78.

19 Vgl. insoweit die Präambel des «Agreement concerning the Adoption of Harmonized Technical United Nations Regulations for Wheeled Vehicles, Equipment and Parts which can be Fitted 
Spezialvorschriften für bestimmte Bauteile oder Emissionen erlassen. ${ }^{20}$ Aufgrund der häufigen und regelmäßigen Anpassungen werden die ECE-Regelungen für besonders geeignet gehalten, den technischen Fortschritt im Bereich des autonomen Fahrens regulatorisch zu erfassen. ${ }^{21}$

Durch den Verweis in Art. 8 Abs. $5^{\text {bis }}$ und Art. 39 Abs. 1 S. 3 WÜ wird die Konformität mit den internationalen technischen Normen als eine zweite Grundlage neben den technischen Bestimmungen des Anhangs 5 für die Wahrung der Anforderungen des WÜ etabliert.

\section{Verweis auf technische Standards in der Typgenehmigung}

Die wesentliche Voraussetzung der Zulassung eines Fahrzeugs zum Straßenverkehr ist, wie dargestellt, die sog. Betriebserlaubnis, mit der insbesondere die generelle Betriebssicherheit eines Fahrzeugs bzw. eines Fahrzeugtyps bestätigt wird. Die Betriebserlaubnis wird in einem vom Verfahren der Zulassung eines konkreten Kraftfahrzeuges zu unterscheidenden, separaten Verfahren erteilt. Dabei ist die auf einen Fahrzeugtyp bezogene Genehmigung, die sog. «Typgenehmigung» oder «Typzulassung», von der auf ein einzelnes Fahrzeug bezogenen Genehmigung, der sog. «Einzelgenehmigung», zu unterscheiden. ${ }^{22}$

and/or be Used on Wheeled Vehicles and the Conditions for Reciprocal Recognition of Approvals Granted on the Basis of these United Nations Regulations» vom 20.10.2017, abrufbar unter: https://www.unece.org/fileadmin/DAM/trans/main/wp29/wp29regs/2017/E-ECE-TRANS505-Rev.3e.pdf(abgerufen am 13.01.2020).

Die Addenda sind abrufbar unter http://www.unece.org/trans/main/wp29/wp29regs.html (abgerufen am 13.01.2020).

21 Siehe etwa LuTZ, in: HILGENDORF/HöTITZSCH/LuTZ, Rechtliche Aspekte automatisierter Fahrzeuge - Beiträge zur 2. Würzburger Tagung zum Technikrecht im Oktober 2014, 2015, S. 33, 47; zu den rechtlichen Anforderungen an eine Änderung ausführlich v. UNGERN-STERNBERG, in: OPPERMAnN/STENDER-VoRWACHS, Autonomes Fahren, 2017, S. 299.

Ebenso aus Schweizer Sicht: Bundesamt für Strassen (ASTRA), Führerlose Fahrzeuge - Rechtlicher Anpassungsbedarf, 22.04.2016, S. 18.

22 Insoweit besteht etwa nach deutschem Recht die Möglichkeit zur Erteilung einer Betriebserlaubnis für Einzelfahrzeuge, vgl. § 21 der Straßenverkehrs-Zulassungs-Ordnung (StVZO). 
In der Praxis ist vor allem die Typgenehmigung (Typzulassung) von Bedeutung, insbesondere die EG-Typgenehmigung auf der Grundlage der Richtlinie 2007/46/EG (Typgenehmigungs-Verfahrensrichtlinie). ${ }^{23}$ Die EG-Typgenehmigung wird durch den Hersteller eines Fahrzeuges bei der nach nationalem Recht zuständigen Behörde beantragt; für das Verfahren enthalten die Art. 6 ff. RL 2007/46/EG Vorgaben, die durch die Mitgliedstaaten in nationales Recht umzusetzen sind. In Deutschland ist insoweit die EGFahrzeuggenehmigungsverordnung (EG-FGV) 24 maßgeblich. Die nach deutschem Recht zuständige Behörde für die Erteilung von EG-Typgenehmigungen ist gemäß § 2 Abs. 1 EG-FGV das Kraftfahrt-Bundesamt (KBA).

Die materiellen Anforderungen an die EU-Typgenehmigung sind in Art.9 Abs. 1 Nr. 1 RL 2007/46/EG sowie Art. 10 in Verbindung mit Anhang IV und Anhang XI der Richtlinie geregelt. Entsprechend verweisen auch die nationalen Umsetzungen auf diese Artikel und die Anhänge (vgl. zum deutschen Recht § 4 Abs. 4 EG-FGV). ${ }^{25}$ Die Anforderungen des Anhangs IV stehen damit im Zentrum der EG-Typzulassung.

Ab dem 1. September 2020 wird die Typgenehmigungsverfahrens-Verordnung ${ }^{26}$ an die Stelle der bisherigen Richtlinie treten, die aus Anlass des «Dieselskandals» eine erhebliche Erweiterung der Befugnisse der Europäischen Kommission im Hinblick auf die Überwachung des Typgenehmigungsverfahrens und die Marktüberwachung mit sich

Richtlinie 2007/46/EG des Europäischen Parlaments und des Rates vom 5. September 2007 zur Schaffung eines Rahmens für die Genehmigung von Kraftfahrzeugen und Kraftfahrzeuganhängern sowie von Systemen, Bauteilen und selbstständigen technischen Einheiten für diese Fahrzeuge, ABl. EU L 263, 1 ff.

Verordnung über die EG-Genehmigung für Kraftfahrzeuge und ihre Anhänger sowie für Systeme, Bauteile und selbstständige technische Einheiten für diese Fahrzeuge vom 3.2.2011, BGBl. I 2011, 126.

$\S 4$ Abs. 4 EG-FGV lautet insoweit auszugsweise: «Die EG-Typgenehmigung darf nur erteilt werden, wenn die Voraussetzungen [...] nach Artikel 8 Absatz 1 der Richtlinie 2007/46/EG vorliegen und nach Artikel 8 Absatz 2 der Richtlinie 2007/46/EG die Erfüllung der spezifischen Bestimmungen der Artikel 9 und 10 sichergestellt ist [...]»

26 Verordnung (EU) 2018/858 des Europäischen Parlaments und des Rates vom 30. Mai 2018 über die Genehmigung und die Marktüberwachung von Kraftfahrzeugen und Kraftfahrzeuganhängern sowie von Systemen, Bauteilen und selbstständigen technischen Einheiten für diese Fahrzeuge, zur Änderung der Verordnungen (EG) Nr. 715/2007 und (EG) Nr. 595/2009 und zur Aufhebung der Richtlinie 2007/46/EG, ABl. EU L 151, S. 1 ff. 
bringt. ${ }^{27}$ Die für diesen Beitrag wesentlichen Bestandteile der Typgenehmigungs-Verfahrensrichtlinie bleiben jedoch unangetastet, insbesondere die materiellen Anforderungen an die Erteilung der Typgenehmigung bleiben unverändert in Art. 5 Abs. 1 und in Art. 29 Abs. 1 Typgenehmigungsverfahrens-VO erhalten. ${ }^{28}$

\section{Koordinierung des WÜ und des europäischen Rechts mit den UN/ECE-Regelungen}

Die Existenz mehrerer Regelungssysteme zur Betriebserlaubnis und zu technischen Anforderungen an die Betriebssicherheit erfordert eine Koordination der Regelungssysteme, um Überregulierung oder gar widersprüchliche Anforderungen zu vermeiden.

Dabei sind zwei Koordinierungsaufgaben zu unterscheiden: Da die Mitgliedstaaten der EU als Vertragsstaaten an die Vorgaben des WÜ gebunden sind, ist insoweit eine Koordinierung des europäischen Rechts mit dem WÜ erforderlich. Unabhängig davon ist eine Koordinierung der Anforderungen des WÜ mit denen des UN/ECE-Übereinkommens geboten.

Diese Aufgaben wurden durch Verweis des EU-Rechts und des WÜ auf die UN/ECERegeln gelöst. Auf der Ebene des WÜ erfolgt die Koordinierung über den neuen Art. 39 Abs. 1 S. 3 WÜ, der unmittelbar auf UN/ECE-Normen verweist und diese als gleichwertig zu den Anforderungen des Anhangs 5 bezeichnet. ${ }^{29}$

Die Richtlinie 2007/46/EG verfolgt ebenfalls ein Konzept der Anerkennung von UN/ECE-Normen als gleichwertig. Rechtstechnisch werden insoweit zwei Instrumente genutzt. Zum einen wird in Anhang IV zur Richtlinie 2007/46/EG, der die wesentlichen technischen Anforderungen enthält, alternativ auf technische Vorschriften und auf UN/ECE-Normen verwiesen. Die UN/ECE-Normen werden also in Anhang IV und damit in das europäische System der Typgenehmigung inkorporiert. Zum anderen sieht

27 Vgl. im Überblick zu den Neuerungen WAGNER/KARAGKOUNI, EuZW 2019, 108 ff.

28 Einzig die bisherige Unterscheidung zwischen «Fahrzeugtypen» und «Fahrzeugtypen mit besonderer Zweckbestimmung» (vgl. Art. 9 Abs. 1 Typgenehmigungsverfahrens-Richtlinie) fällt nunmehr weg. 
die Richtlinie die Anerkennung von Genehmigungen nach UN/ECE-Regeln als gleichwertig zu einer EG-Typgenehmigung vor (vgl. Teil II des Anhangs IV Richtlinie 2007/46/EG). Das System der alternativen Inkorporation von UN/ECE-Regelungen oder der Anerkennung als gleichwertig wird durch die TypgenehmigungsverfahrensVO beibehalten, die in dem neuen Anhang II (ersetzt die bisherigen Anhänge IV und XI der Richtlinie) in Teil I und Teil II eine entsprechende Unterscheidung trifft.

\section{Zwischenergebnis}

Damit ergibt sich, dass die gegenwärtige Regelung der Betriebserlaubnis von Kraftfahrzeugen als wesentliche materielle Anforderung für die Zulassung von Kraftfahrzeugen zum Straßenverkehr auf einer dynamischen Verweisung auf technische Standards beruht, die sowohl im europäischen Recht der Typgenehmigung als auch im Wiener Übereinkommen maßgeblich ist.

Im Zuge der jüngsten Änderung des WÜ und der europäischen Typgenehmigungsverfahrens-Richtlinie wurde dabei eine bemerkenswerte Dynamisierung und Harmonisierung dieses Verweises herbeigeführt. Zum einen sind die Verweise, soweit sie die sogenannten UN/ECE-Normen betreffen, vollständig dynamisiert und ausgelagert: Während der traditionelle Verweis des Art. 39 WÜ auf den Anhang 5 innerhalb des WÜ-Systems verbleibt und jeweils eine Änderung nach dem Abstimmungssystem des WÜ verlangt, vertraut das WÜ durch den Verweis auf die UN/ECE-Regeln nunmehr alternativ auch auf die technischen Normen des Fahrzeugteileübereinkommens. Eine parallele Regelung ergibt sich bei den Anforderungen an die europäische Typgenehmigung, die nunmehr ebenfalls auf das UN/ECESystem verweisen.

Damit hat sich in jüngster Zeit, jedenfalls für automatisierte Fahrzeuge, eine sehr bemerkenswerte Verlagerung und Vereinheitlichung der Festlegung technischer Anforderungen ergeben, die im Ergebnis die Normung auf die UN/ECE-Regeln des Fahrzeugteileabkommens verlagert.

Der Gesetzgeber legt also die Gestaltung der technischen Anforderungen an die Sicherheit von Fahrzeugen weitgehend in die Hände der internationalen technischen Normung. 


\section{STRUKTURELLE HERAUSFORDERUNGEN FÜR DAS RECHT DURCH KÜNSTLICHE INTELLIGENZ}

Die bekannten Instrumente der Harmonisierung und des Verweises auf technische Standards werden, wie dargestellt, auch für die Regulierung des automatisierten Fahrens genutzt. Die daran anschließende, hier im Fokus stehende Frage geht dahin, ob diese Instrumente ausreichen oder strukturelle Änderungen des Rechtsrahmens erforderlich sind.

\section{Autonomes Verhalten von Fahrzeugen als Herausforderung}

Eine wesentliche Besonderheit von Systemen, die aufgrund künstlicher Intelligenz eigenständig Entscheidungen über die Zielerreichung treffen und diese umsetzen, besteht darin, dass das konkrete Verhalten der Maschine nicht unmittelbar auf eine menschliche Entscheidung zurückgeführt werden kann. Im Fall des automatisierten Fahrens wird das konkrete Fahrverhalten - technisch: die Längs- und Quersteuerung des Fahrzeugs auf der Straße - nicht mehr von einem Menschen (dem Fahrer), sondern vom Fahrzeug selbst kontrolliert.

Diese Automatisierung ist für das Recht eine Herausforderung, da traditionell die Steuerung von Verhalten als eine ausschließlich menschliche Tätigkeit verstanden und entsprechend der rechtlichen Regelung zugrunde gelegt wurde. Dies gilt uneingeschränkt für das Straßenverkehrsrecht; das Wiener Übereinkommen belegt dies sehr anschaulich: Die im dritten Kapitel des WÜ zusammengefassten Regeln über die Zulassung von Fahrzeugen beziehen sich auf technische Eigenschaften des Fahrzeugs, die insoweit maßgeblichen Anforderungen werden durch die in Anhang 5 referenzierten technischen Standards und nunmehr - alternativ - durch die UN/ECE-Regeln festgelegt. Das Verhalten im Straßenverkehr hingegen wird in Kapitel II adressiert, das die Verkehrsregeln enthält. Im Zentrum steht insoweit der Fahrer des Fahrzeuges, der traditionell als menschlicher Fahrer verstanden wird.

\section{Das traditionelle Erfordernis eines menschlichen Fahrzeugführers}

Die Existenz eines Fahrzeugführers und die Anforderungen an dessen Verhalten sind im Wiener Übereinkommen von zentraler Bedeutung. So muss nach Art. 8 
Abs. 1 WÜ jedes Fahrzeug einen Fahrzeugführer haben. Weiterhin enthält das Übereinkommen zahlreiche Verkehrsregeln, die sich in Gestalt von Verhaltensanforderungen an den Kraftfahrzeugführer richten. Die Anforderung des Art. 8 Abs. 1 WÜ hat entscheidende Bedeutung für die Teilnahme hochautomatisierter Fahrzeuge am Straßenverkehr. Der Begriff des «Fahrzeugführers» wird traditionell dahin verstanden, dass ein menschlicher Fahrer erforderlich sei (vgl. Art. 1 lit. v WÜ). ${ }^{30}$ Weiterhin sieht Art. 8 Abs. 5 WÜ vor, dass jeder Führer sein Fahrzeug dauerhaft beherrschen können muss.

Beide Vorgaben zusammengenommen schließen nach herrschender Lesart ein hochund vollautomatisiertes Fahren der SAE-Stufen 3-5 aus. ${ }^{31}$ Nicht ausgeschlossen sind jedoch Fahrassistenzsysteme, die den menschlichen Fahrer unterstützen, aber nicht ersetzen. ${ }^{32}$ Als Grenze wird insoweit gesehen, dass der menschliche Fahrer jederzeit die volle Kontrolle jedenfalls in der Weise hat, dass er automatisierte Fahrfunktionen übersteuern kann. ${ }^{33}$

Zur Beseitigung dieses Hindernisses für die Teilnahme von hoch- und vollautomatisierten Fahrzeugen am Straßenverkehr wurde, wie dargestellt (vgl. C.I.2.), in der Revision des WÜ 2016 der neue Art. 8 Abs. $5^{\text {bis }}$ WÜ eingefügt. ${ }^{34}$ Art. 8 Abs. $5^{\text {bis } W U ̈ ~ i s t ~}$ derzeit die zentrale rechtliche Grundlage für die Verwendung von automatisierten Fahrfunktionen im Verkehr und enthält entscheidende Änderungen gegenüber dem bisherigen System des WÜ.

Die Vorschrift enthält zwei Sätze mit ganz unterschiedlichem Gehalt: Satz 1 verweist auf technische Anforderungen. Danach ist das Erfordernis des Abs. 5 - ebenso Art. 13

JÄNICH/SCHRADER/RECK, NZV 2015, 313, 314, 315; LUTZ/TANG/LIENKAMP, NZV 2013, 57, 58.

ARZT/Ruth-SCHUMACHER, NZV 2017, 57, 61; v. BodungEN/HOFFMANN, SVR 2016, 41, 43; KüTÜKMARKENDORF/ESSERS, MMR 2016, 22, 23; LUTZ/TANG/LIENKAMP, NZV 2013, 57, 58; vgl. auch FLECK/THOMAS, NJOZ 2015, 1393, 1395.

ALBRECHT, SVR 2005, 373 f.; JouRdAN/MATSCHI, NZV 2015, 26, 29; KÜTÜK-MARKENDORF/ESSERS, MMR 2016, 22, 23.

ALBRECHT, SVR 2005, 373 f.; JouRdAN/MATSCHI, NZV 2015, 26, 29; KÜTÜK-MARKENDORF/ESSERS, MMR 2016, 22, 23. DIES., SVR 2016, 41 ff. sowie DIES., SVR 2016, 93, 94 f.; JÄNICH/SCHRADER/RECK, NZV 2015, 313, 318; LUTZ, DAR 2014, 446 ff.; DERS., NJW 2015, 119, 122 ff.; DERS., DAR 2016, 55 ff. 
Abs. 1 WÜ - erfüllt, wenn die Systemelemente den Vorgaben der internationalen Rechtsvorschriften, konkret der UN/ECE-Standards oder den Vorgaben des GTR-Übereinkommens, ${ }^{35}$ entsprechen. Nach Satz 2 verstoßen automatisierte Systeme nicht gegen die Anforderungen des Art. 8 Abs. 5 WÜ, wenn die Fahrzeugsysteme vom Fahrzeugführer übersteuert oder deaktiviert werden können. ${ }^{36}$ Beide Bestandteile des Absatzes sind für das automatisierte Fahren gleichermaßen wichtig, wenngleich auf ganz unterschiedliche Weise.

Während der Verweis auf technische Standards das bereits erörterte Konzept des Vertrauens auf internationale technische Normung umsetzt, trifft Satz 2 eine entscheidende regulatorische Weichenstellung, indem die Möglichkeit des Übersteuerns ohne Bezugnahme auf die Eigenschaften des technischen Systems als ausreichende Fahrzeugbeherrschung angesehen wird. Die Teilnahme mit hoch- und vollautomatisierten Fahrzeugen am Straßenverkehr ist danach, auch ohne die durch Art. 8 Abs. $5^{\text {bis }}$ Satz 1 WÜ in Bezug genommenen UN/ECE-Normen zu erfüllen, zulässig, soweit die jederzeitige Übernahmemöglichkeit durch einen menschlichen Fahrer besteht.

Diese Regelung schärft zum einen den Begriff der Fahrzeugbeherrschung, die offensichtlich bei jederzeitiger Übernahmemöglichkeit noch gegeben sein soll, und zum anderen wird die Möglichkeit geschaffen, automatisiertes Fahren jedenfalls unter dieser Prämisse zuzulassen. Dies ist eine wesentliche Grundlage für den gegenwärtigen Stand der technischen Entwicklung im Hinblick auf das automatisierte Fahren und schafft eine Möglichkeit, den Betrieb von hochautomatisierten Fahrzeugen - wenngleich unter Einschränkungen - zu realisieren.

Eine dauerhafte Lösung für hoch- und vollautomatisiertes Fahren bietet dieser Ansatz indes nicht. Entsprechend ist nicht verwunderlich, dass sich zahlreiche Fragen um die Anforderungen an die Möglichkeit zur jederzeitigen Übernahme der Fahrzeugsteuerung ranken, die keineswegs einfach zu lösen sind.

35 Zur Bedeutung des Verweises oben C.I.2.

36 Gemäß Art. 8 Abs. 5 WÜ und Art. 13 Abs. 1 WÜ muss der Führer das Fahrzeug dauernd «beherrschen», um den Sorgfaltspflichten nachkommen zu können und ständig in der Lage zu sein, die ihm obliegenden Fahrbewegungen auszuführen. 


\section{Künstliche Intelligenz als Herausforderung für das System der UN/ECE-Regelungen}

Die Änderung des WÜ durch den neuen Art. 8 Abs. $5^{\text {bis }}$ vertraut, wie dargestellt, hinsichtlich der Anforderungen an den automatisierten Betrieb des Fahrzeugs auf technische Standards. Die alternative Bezugnahme auf die Übernahme, die als Fortdauer der menschlichen Fahrzeugsteuerung angesehen werden kann, ist im Kernbereich des automatisierten Fahrens und damit für die Fahrzeuge der Automatisierungsstufen 4 und 5 keine ausreichende Maßgabe, sodass es insoweit beim Verweis auf die Erfüllung technischer Standards verbleibt.

Bereits bei den Beratungen um die Änderung des WÜ war umstritten, ob die beschlossene Änderung ausreicht. So hatten etwa Belgien und Schweden ein Konzept vorgeschlagen, das auf die Anerkennung einer maschinellen Steuerung als «Fahrzeugführer» durch eine Änderung des Art. 1 lit. v) WÜ abzielte, konnten sich damit jedoch in der Arbeitsgruppe nicht durchsetzen. ${ }^{37}$

In Bezug auf die in Kraft getretene Änderung des WÜ wird als problematisch angesehen, dass Art. 8 Abs. $5^{\text {bis }}$ WÜ einzig auf die Art. 8 Abs. 5 und Art. 13 Abs. 1 WÜ verweist, nicht aber auf andere Vorschriften, die Verhaltensanforderungen für den Fahrzeugführer festlegen (siehe etwa Art. 8 Abs. 6, Art. 11 Abs. 4 WÜ). ${ }^{38}$ Dieses Problem hat auch das schweizerische Bundesamt für Strassen (ASTRA) in einer Studie zum führerlosen Fahren aus dem Jahr 2016 erkannt und aufgrund der (noch) bestehenden technischen Hürden als einstweilen nicht klärungsbedürftig angesehen. ${ }^{39}$ Die Studie verweist insoweit darauf, dass es einer entsprechenden UN/ECE-

37 Siehe Economic Commission for Europe/Working Party on Road Traffic Safety, Seventieth session (Geneva, 23-26 March 2015), Informal Document No. 2, S. 6 f. (abrufbar unter: http://www.unece.org/fileadmin/DAM/trans/doc/2015/wp1/ECE-TRANS-WP1-INT-2e.pdf (abgerufen am 13.01.2020)); zu diesem Diskussionspapier ausführlich V. BoDUNGEN/HoFFMANN, NZV 2015, 521 ff.; V. UnGERN-STERNBERG, in: OPPERMANN/STENDER-VORWACHS (Hrsg.), Autonomes Fahren, 2017, S. 310 f.

LuTZ, in: HILGENDORF/HöTITZSCH/LuTZ (Hrsg.), Rechtliche Aspekte automatisierter Fahrzeuge Beiträge zur 2. Würzburger Tagung zum Technikrecht im Oktober 2014, 2015, S. 33, 40 ff.

39 BundeSAmT Für StRASSEn (ASTRA), Führerlose Fahrzeuge - Rechtlicher Anpassungsbedarf, 22.04.2016, S. $18 \mathrm{f}$. 
Regelung für nicht abschaltbare oder nicht übersteuerbare Systeme bedürfe, die gewährleiste, dass die Pflichten des Fahrzeugführers dauerhaft durch das System erfüllt werden können.

Diese Fragestellung wurde auch in der deutschen Literatur untersucht. Insoweit wird vorgeschlagen, auch solche Fahrzeuge als mit den Vorgaben des WÜ vereinbar anzusehen, in denen automatisierte Fahrzeugsysteme zum Einsatz gelangen, die nicht zu überwachen und/oder nicht übersteuerbar sind.40 Dogmatisch wird dies etwa damit begründet, dass es sich bei der Regelung des Art. 8 Abs. $5^{\text {bis } W U ̈ ~ u m ~}$ eine spezielle und damit vorrangige Vorschrift im Hinblick auf die Zulässigkeit des Betriebs von hoch- und vollautomatisierten Fahrzeugen handelt. ${ }^{41}$ Teilweise wird auch eine teleologische Reduktion der - im Grundsatz weiterhin anwendbaren ${ }^{42}$ Verhaltensvorschriften, die auf menschliche Fahrer zugeschnitten sind, vorgeschlagen. ${ }^{43}$

Zur Umsetzung des neuen Art. 8 Abs. $5^{\text {bis } W U ̈ ~ h a t ~ d e r ~ d e u t s c h e ~ G e s e t z g e b e r ~ d u r c h ~ d a s ~}$ Achte Gesetz zur Änderung des Straßenverkehrsgesetzes vom 16.6.2017, ${ }^{44}$ das am 21.6.2017 in Kraft getreten ist, die neuen $\S$ 1a und § $1 \mathrm{~b}$ in das Straßenverkehrsgesetz aufgenommen, die für Fahrzeuge mit hoch- und vollautomatisierten Fahrfunktionen weitere, spezielle Vorgaben im Hinblick auf den Betrieb dieser Fahrzeuge im Straßenverkehr festlegen.

So ist gemäß § 1a Abs. 1 StVG der Betrieb eines Kraftfahrzeugs mit hoch- bzw. vollautomatisierter Fahrfunktion nur zulässig, wenn diese Funktion bestimmungsgemäß verwendet wird. In der Literatur wird an dieser Voraussetzung mitunter kritisiert, dass Hersteller künftig als «Ersatzgesetzgeber» fungieren, indem diese den «bestimmungs-

40 LUTZ, NJW 2015, 119, 123 f.; DERS., DAR 2016, 55, 55 f.; DERS., in: HILGENDORF/HöTITZSCH/LuTZ (Hrsg.), Rechtliche Aspekte automatisierter Fahrzeuge - Beiträge zur 2. Würzburger Tagung zum Technikrecht im Oktober 2014, 2015, S. 33, 40 ff.; V. UNGERN-STERNBERG, in: OPPERMANN/STENDER-VORWACHS (Hrsg.), Autonomes Fahren, 2017, S. 308 f.

41 V. Ungern-Sternberg, in: Oppermann/Stender-Vorwachs (Hrsg.), Autonomes Fahren, 2017, S. $308 \mathrm{f}$.

42 V. Bodungen/HoffmanN, SVR 2016, 41, $45 \mathrm{ff}$.

43 V. Bodungen/HofFmanN, SVR 2016, 93, $94 \mathrm{f}$.

44 BGBl. I 2017, S. 1648. 
gemäßen Gebrauch» von hoch- und vollautomatisierten Fahrzeugen definieren und damit den Maßstab für deren Benutzung setzen. ${ }^{45}$ Weitere Voraussetzung ist, wie aus § 1a Abs. 3 StVG folgt, dass das Fahrzeug die in § 1a Abs. 2 StVG genannten technischfunktionalen Mindestanforderungen erfüllt.

Das Konzept des Art. 8 Abs. $5^{\text {bis } W U ̈ ~ i s t ~ i n ~ § ~ 1 a ~ S t V G ~ u m g e s e t z t . ~ N a c h ~ § ~ 1 a ~ A b s . ~} 4$ StVG ist Fahrzeugführer auch derjenige, der eine hoch- oder vollautomatisierte Fahrfunktion aktiviert. Fahrzeuge mit hoch- oder vollautomatisierten Fahrfunktionen sind nach § 1a Abs. 2 S. 1 Nr. 3 StVG zulässig, wenn diese jederzeit durch den Fahrzeugführer manuell übersteuerbar oder deaktivierbar sind. Dies stimmt mit dem Konzept des WÜ überein, da dieses - wie dargestellt - den passiven Fahrer nach Art. 8 Abs. $5^{\text {bis }}$ WÜ zulässt, wenn das Fahrzeug den entsprechenden technischen Vorschriften genügt. § $1 \mathrm{~b}$ StVG regelt Pflichten des Fahrers im Zusammenhang mit der jederzeitigen Möglichkeit zur Übernahme der Fahrzeugsteuerung. Mit diesem Grundkonzept bleibt der deutsche Gesetzgeber ganz im System des Art. 8 Abs. $5^{\text {bis } W U ̈ . ~}$

Das Konzept der jederzeitigen Übernahmemöglichkeit im deutschen StVG wirft erhebliche Folgefragen auf, die derzeit kritisch diskutiert werden. ${ }^{46}$ Problematisch ist insoweit etwa die Bestimmung des dem Fahrzeugführer abverlangten Verhaltens im Hinblick auf Übernahmebereitschaft, Reaktionszeiten und sonstige Pflichten. ${ }^{47}$ Während der deutsche Bundesrat im Gesetzgebungsverfahren noch eine Reaktionszeit von 1,5 bis 2 Sekunden zuzüglich eines Sicherheitszuschlags gefordert hatte, ${ }^{48}$ wird in der Literatur davon ausgegangen, dass dem Fahrzeugführer keine «Schrecksekunde» im Sinne einer Prüf- oder Bedenkzeit zugebilligt werde, ${ }^{49}$ sondern der Fahrzeugführer unter Berücksichtigung der konkreten Situation so schnell wie möglich die Steuerung übernehmen muss. ${ }^{50}$

LÜDEMANN/SUTTER/VOGELPOHL, NZV 2018, 411, 412; weniger kritisch insoweit KöNIG, NZV 2017, $123,125$.

Hierzu im Überblick v. BodUngEn/HofFMAnN, NZV 2018, 97 ff.; v. KALER/WIESER, NVwZ 2018, 369 ff.; KÖNIG, NZV 2017, 123 ff.; LÜDEMANN/SUTTER/VOGELPOHL, NZV 2018, 411 ff.; SCHIRMER, NZV 2017, $253 \mathrm{ff}$.

Siehe etwa Buck-Heeb/Dieckmann, NZV 2019, 113 ff.; Greger, NZV 2018, 1 ff.; LÜDEmanN/ SUTTER/VOGELPOHL, NZV 2018, $411 \mathrm{ff}$.

Stellungnahme des Bundesrates zu § 1b StVG, BT-Drs. 18/11534, S. 4.

GREGER, NZV 2018, 1, 2. 


\section{Herstellererklärung vs. Typgenehmigung?}

Die aktuelle Diskussion zur Zulassungsfähigkeit hoch- und vollautomatisierter Fahrzeuge verweist, wie dargestellt, auf die UN/ECE-Regeln und stattet das System der UN/ECE somit mit einem erheblichen Vertrauensvorschuss aus. Die erforderliche Weiterentwicklung der UN/ECE-Regeln muss aber erhebliche Herausforderungen bewältigen. Der neue § 1a Abs. 2 S. 1 StVG nennt die Herausforderungen deutlich: Das Fahrzeug muss hiernach über eine technische Ausrüstung verfügen,

- die zur Bewältigung der Fahraufgabe - einschließlich Längs- und Querführung das jeweilige Kraftfahrzeug nach Aktivierung steuern (Fahrzeugsteuerung) kann (Nr. 1),

- die in der Lage ist, während der hoch- oder vollautomatisierten Fahrzeugsteuerung den an die Fahrzeugführung gerichteten Verkehrsvorschriften zu entsprechen (Nr. 2),

- die jederzeit durch den Fahrzeugführer manuell übersteuerbar oder deaktivierbar ist (Nr. 3),

- die die Erforderlichkeit der eigenhändigen Fahrzeugsteuerung durch den Fahrzeugführer erkennen kann (Nr. 4),

- die dem Fahrzeugführer das Erfordernis der eigenhändigen Fahrzeugsteuerung mit ausreichender Zeitreserve vor der Abgabe der Fahrzeugsteuerung an den Fahrzeugführer optisch, akustisch, taktil oder sonst wahrnehmbar anzeigen kann (Nr. 5) und

- die auf eine der Systembeschreibung zuwiderlaufende Verwendung hinweist (Nr. 6).

Es besteht kein Zweifel daran, dass diese Eignung idealerweise im Rahmen der Typgenehmigung festzustellen ist. Es müssen also entsprechende Regeln für die Zulassung ausgearbeitet werden. Das dürfte im Hinblick auf die genannten Aspekte keine einfache Aufgabe sein, da es nicht um statische technische Eigenschaften geht.

Erforderlich ist also, dass im Rahmen der Typgenehmigung eine verlässliche Prognose über das Fahrverhalten getroffen werden kann, das wiederum entscheidend von der 
Verkehrssituation abhängt. Es wird also erforderlich sein, das Prüfsystem der Zulassung wesentlich zu erweitern.

Darüber hinaus stellt sich die Frage, ob eine Prüfung im Rahmen der Typgenehmigung ausreichend, geschweige denn ohne weiteres möglich ist. Der deutsche Gesetzgeber bezweifelt dies offensichtlich, denn er hat sich bei der Anpassung des StVG an das geänderte WÜ auf die Umsetzung des Art. 8 Abs. $5^{\text {bis }}$ WÜ beschränkt und mit dem neuen $\S$ 1a Abs. 2 StVG eine Regelung in das StVG eingefügt, die technische Anforderungen an das Fahrzeug - die eher im Bereich der Zulassung zu erwarten gewesen wären - auf den Betrieb des Fahrzeugs bezieht.

Nach § 1a Abs. 1 i.V.m. Abs. 2 StVG ist der Betrieb eines hoch- oder vollautomatisierten Fahrzeuges zulässig, wenn das Fahrzeug die Anforderungen nach Abs. 2 S. 1 Nr. 1-6 erfüllt. Der Betrieb von hoch- oder vollautomatisierten Fahrzeugen setzt nach § 1a Abs. 3 StVG zudem voraus, dass diese zugelassen sind und den maßgeblichen «internationalen Vorschriften» entsprechen oder über eine EG-Typgenehmigung verfügen. ${ }^{51}$ Dies macht deutlich, dass die Vorgaben des Abs. 2 keine Anforderungen an die Zulassung darstellen.

§ 1a Abs. 2 StVG enthält damit - außerhalb der Fahrzeugzulassung - in der Sache Konstruktionsvorgaben, die sich an Fahrzeughersteller richten.52 Dies wird besonders deutlich anhand des § 1a Abs. 2 S. 2 StVG, der wie folgt lautet: «Der Hersteller eines solchen

$51 \S 1$ a Abs. 3 StVG lautet:

«(3) Die vorstehenden Absätze sind nur auf solche Fahrzeuge anzuwenden, die nach $\S 1 \mathrm{Ab}$ satz 1 zugelassen sind, den in Absatz 2 Satz 1 enthaltenen Vorgaben entsprechen und deren hoch- oder vollautomatisierte Fahrfunktionen

1. in internationalen, im Geltungsbereich dieses Gesetzes anzuwendenden Vorschriften beschrieben sind und diesen entsprechen oder

2. eine Typgenehmigung gemäß Artikel 20 der Richtlinie 2007/46/EG des Europäischen Parlaments und des Rates vom 5. September 2007 zur Schaffung eines Rahmens für die Genehmigung von Kraftfahrzeugen und Kraftfahrzeuganhängern sowie von Systemen, Bauteilen und selbstständigen technischen Einheiten für diese Fahrzeuge (Rahmenrichtlinie) (ABl. L 263 vom 9.10.2007, S. 1) erteilt bekommen haben.» 
Kraftfahrzeugs hat in der Systembeschreibung verbindlich zu erklären, dass das Fahrzeug den Voraussetzungen des Satzes 1 entspricht.»

Das Konzept der «verbindlichen Erklärung» ist im Gesetz nicht näher ausgestaltet. Für die hier interessierende Fragestellung aber ist klar, dass der deutsche Gesetzgeber spezielle Anforderungen an hoch- und vollautomatisierte Fahrzeuge stellt, die rechtstechnisch nicht an die Zulassung, sondern an den Betrieb des Fahrzeugs geknüpft werden. Ob dies mit den Vorgaben des WÜ im Einklang steht, wird bisher nicht im Einzelnen diskutiert. In der deutschen Literatur wird bisher davon ausgegangen, dass die Ergänzungen des StVG im Jahr 2017 mit den Vorgaben des WÜ im Einklang stehen, insbesondere weil insoweit am Konzept des menschlichen Fahrers im Grundsatz festgehalten wird. ${ }^{53}$

Allerdings wirft die im Gesetz genannte Herstellererklärung etliche Fragen auf. Insbesondere knüpft das Gesetz keine ausdrücklichen Rechtsfolgen an das Fehlen der Erklärung. Aus der Verbindung mit $\S$ 1a Abs. 1 StVG dürfte wohl zu schließen sein, dass das Fahrzeug ohne eine solche Erklärung nicht betrieben werden darf. Ob dieses Erfordernis auch für im Ausland zugelassene Fahrzeuge gilt, macht das Gesetz nicht deutlich. Da das Erfordernis der Herstellererklärung im WÜ nicht enthalten ist, wird man dies jedenfalls nicht ohne weiteres annehmen können, da der Gesetzgeber sonst gegen das WÜ verstieße. Ebenso ist unklar, welche Folgen die Unrichtigkeit einer Herstellererklärung auslöst. Auch insoweit schweigt das Gesetz.

Das Konzept der Herstellererklärung ist also jedenfalls noch nicht ausgereift und kann derzeit nicht alle Fragen beantworten. Der deutsche Alleingang macht aber deutlich, dass die schon bei Änderung des WÜ diskutierte Frage, wie die spezifischen Herausforderungen «autonomen» Verhaltens - mithin das Wesen des automatisierten Fahrens bewältigt werden können, durch den derzeitigen Rechtsrahmen noch nicht befriedigend beantwortet wird. 


\section{Notwendigkeit der Fortentwicklung des Rechtsrahmens für automatisierte Fahrzeuge}

Das WÜ vertraut, wie dargestellt, in Bezug auf die Sicherstellung der Betriebssicherheit von Fahrzeugen entscheidend auf die Betriebserlaubnis. Wie dargestellt, ist aber fraglich, ob der bestehende Rahmen der Betriebserlaubnis zur Gewährleistung der Betriebssicherheit ausreichend oder für KI-Anwendungen überhaupt geeignet ist.

Eine zentrale Herausforderung besteht, wie gesagt, darin, im Rahmen der Prüfung der Betriebssicherheit, also im Verfahren zur Erteilung der Betriebserlaubnis, eine Prognose hinsichtlich des Verhaltens des Fahrzeugs in ganz unterschiedlichen Verkehrssituationen zu erstellen. Diese Herausforderung stellt das System der Betriebserlaubnis als solches nicht in Frage. Es sind aber weitere Maßnahmen erforderlich, die durch das Konzept der Betriebserlaubnis nicht abgedeckt werden.

Dies betrifft im Wesentlichen Aspekte der Updates und Weiterentwicklung der Systeme. Hoch- und vollautomatisierte Fahrzeuge werden nach allgemeiner Erwartung regelmäßig Updates erhalten. Dabei sind Updates von Informationen, insbesondere Karteneinträge, von Updates der Systeme, etwa neuer Software zur Fahrzeugsteuerung, zu unterscheiden.

Bei Updates von Karteninformationen und sonstigen Informationen, die laufend erneuert werden, ist im Rahmen der Betriebserlaubnis sicherzustellen, dass ein adäquater Aktualisierungsmechanismus besteht. Darüber hinaus wird man aber zu fordern haben, dass die Nutzung des konkreten Fahrzeugs von der Aktualität der Karteninformation abhängig gemacht wird. Dies wäre, ähnlich wie in § 1 b des deutschen StVG geregelt, im WÜ oder ggf. in den nationalen Verkehrsordnungen zu regeln.

Jedenfalls bei Updates von Software stellt sich die Frage nach der Fortdauer der Betriebserlaubnis. Nach geltendem Recht bezieht sich die Betriebserlaubnis auf den Fahrzeugtyp (Typgenehmigung) oder das Fahrzeug zum Zeitpunkt der Zulassung, sie ist also statisch.

Eine dynamische Betriebserlaubnis, die künftige Änderungen des Fahrzeugs einschließt, ist in den bisherigen Vorschriften zur Erteilung von Betriebserlaubnissen 
nicht vorgesehen. Dies bedeutet, dass bei einer Änderung jeweils eine neue Betriebserlaubnis oder eine Änderung der bestehenden Betriebserlaubnis erforderlich ist (vgl. für das deutsche Recht $§ 5$ EG-FGV). Dieses System bedarf daher der Weiterentwicklung in Bezug auf die Besonderheiten von hoch- und vollautomatisierten Fahrzeugen und dem damit verbundenen Bedarf an der Anpassung und Fortentwicklung der Fahrzeuge.

Weitere Herausforderungen bestehen in Bezug auf die Sicherstellung der Aufbringung von Updates auf das einzelne Fahrzeug. Auch insoweit muss sichergestellt sein, dass die am Verkehr teilnehmenden Fahrzeuge jeweils über die aktuelle Software verfügen, soweit dies aus Gründen der Betriebssicherheit erforderlich ist.

Diese Herausforderungen sind jedenfalls im System des WÜ nicht gelöst. Auch das deutsche StVG löst mit den neu eingefügten §§ 1a, b StVG letztlich nur Teilaspekte dieser Aufgaben.

\section{E. FAZIT}

Die Teilnahme mit voll- und hochautomatisierten Fahrzeugen am Straßenverkehr stellt erhebliche Herausforderungen an das geltende Straßenverkehrsrecht, die durch die Änderung des WÜ von 2016 noch nicht bewältigt sind. Vielmehr sind voraussichtlich noch erhebliche Anpassungen sowohl im WÜ als auch in den nationalen Rechtsordnungen erforderlich.

Im Kern der Herausforderungen steht die Frage, ob die Betriebssicherheit von Fahrzeugen weiterhin ausschließlich durch das Konzept der Betriebserlaubnis erfolgen soll, oder - wie es im deutschen Recht anklingt - durch alternative Konzepte. In jedem Fall besteht Bedarf zur Fortentwicklung des Rechts der Betriebserlaubnis, das auf die Prognose des auch von der Verkehrssituation abhängigen Fahrverhaltens einzustellen ist. Unabhängig davon dürften weitere Maßnahmen, etwa ein System zum Monitoring der Betriebssicherheit, erforderlich sein, die im gegenwärtigen Regelungskonzept des WÜ freilich nicht angelegt sind. 
In allgemeiner Hinsicht zeigt sich die zentrale Bedeutung des Anliegens von Erich Schweighofer, den die Interaktion von Mensch und Maschine und die Frage nach einem internationalen Rahmen für Technologie stets interessiert hat: Die Gestaltung eines angemessenen Rechtsrahmens für neue Technologien bedarf eines interdisziplinären Ansatzes und eines Verständnisses der Technik durch den Gesetzgeber. 